\title{
TERENCE KELLY
}

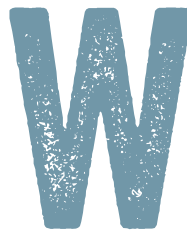

elcome back to Drill Bits, the new column about programming. This second installment explains how decentralized computing relates to distributed computing and parallel computing, describes decentralized solutions to commercially important problems, and provides working example code. To be concrete, l'll begin with practical wireless networking problems; later l'll apply the solutions in other domains.

A decentralized method employs several distinct computations that consume separate inputs and emit separate outputs; the outputs collectively constitute a solution to a given problem. Crucially, no computation accesses all of the inputs required to solve the overall problem. In interesting cases, a change to a single input may change many of the outputs. Computations may, but need not, execute simultaneously andlor in different places; in other words, decentralization is orthogonal to parallelism and distribution.

Practical examples illustrate these distinctions: Internet routing algorithms are inherently distributed because routers are physically dispersed, and each computes its own routes using information gathered from others. The "link state" family of distributed routing algorithms, however, is the antithesis of decentralized because every router assembles a complete map of the 
topology to compute routes. ${ }^{8}$ By contrast, the wireless networking problems considered here admit truly decentralized solutions involving only local computations upon local data.

Software and patterns that support distribution can facilitate decentralization, but the former do not define the latter. For example, a decentralized design may freely leverage old-fashioned remote procedure calls, ${ }^{10}$ triedand-true service-oriented architecture, ${ }^{1}$ or newfangled microservices. ${ }^{4}$ The essence of decentralized computing, however, is not infrastructure but rather ignorance: No entity amasses enough information to solve the problem on its own. Each participant sees a piece of the overall input and generates a piece of the output; in the end everything works out just so, as if a single all-knowing solver were at work.

Why decentralize? Sometimes the inputs for a given problem are inherently scattered, and collecting them would be a bother. Privacy concerns recommend decentralization when entrusting a single entity with all relevant information is feasible yet unwise; just as striving for efficiency reduces effort, ${ }^{3}$ striving for decentralization reduces disclosure. Designing decentralized algorithms can yield insights that improve parallel and distributed algorithms. Most importantly, decentralized methods are sometimes the best way to solve practical problems.

\section{MOBILE WIRELESS PROBLEMS AND SOLUTIONS}

Wireless networks, particularly for mobile devices, are more challenging than networks of utility-powered computers linked by fixed wires. Mobile devices have 
limited energy storage. They move around, so their opportunities for mutual aid and mutual annoyance fluctuate. Devices may be owned and operated by different parties, with no obvious single authority to dictate their behavior.

Figure 1 depicts two thorny problems. Several small devices must communicate with a distant base station. Some pairs of small devices are within close range of one another. If all of the devices talk to the base directly, as in figure 1 [a], then they may consume excessive power andlor jam one another.

A stream of patents spanning two decades underscores the commercial importance of these problems. (Patent applications can cost tens of thousands of dollars in fees and demand years of intermittent focused attention from well-paid engineers and lawyers. Seldom does anyone

\section{FIGURE 1: WIRELESS NETWORKING PROBLEMS \& SOLUTIONS}

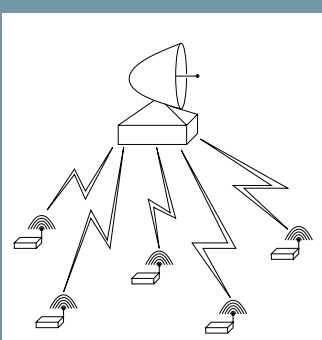

(a)

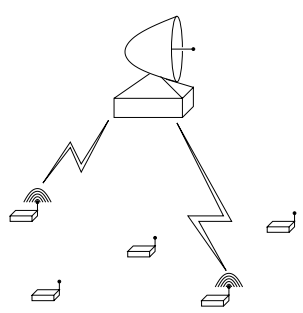

(b)

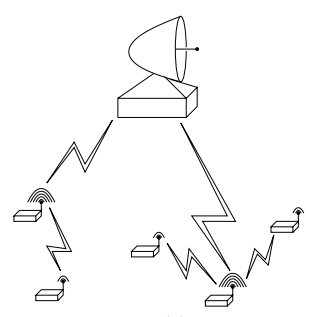

(c) 
squander such time, money, and sustained commitment on unimportant problems or unworkable solutions.]

Let's consider three of these patents. Two prevent mutual jamming by selecting a subset of devices permitted to communicate with the base station simultaneously, as in figure 1(b); no two devices in the subset are close enough to jam one another. ${ }^{5,6} \mathrm{~A}$ third patent reduces overall power consumption by selecting a subset of representative devices to communicate with the base on behalf of nearby peers, as in figure 1[c]; every device is either a representative or is within range of one. ${ }^{9}$ To account for movement of the devices and to spread benefits or burdens evenly across them, each of the three schemes periodically chooses a new subset.

The remarkable thing about these approaches, and other practical solutions to related wireless networking problems, is that they all select subsets of devices the same way. They all compute an MIS (maximal independent set] of devices based on communication/interference adjacencies: a subset of devices such that no two in the MIS are adjacent, and every device outside the MIS is adjacent to at least one that is in it. ${ }^{14} \mathrm{~A}$ given topology may have more than one MIS. In the following topology, for example, the outer circle surrounding the hub constitutes one MIS, and the hub alone is another.

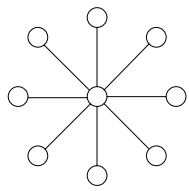

Sometimes you may prefer some MISes over others le.g., based on the number of devices selected or their 
characteristics]. Often, however, any MIS will suffice, as is the case in several wireless networking applications.

A central solver with full knowledge of the network topology can easily compute an MIS: While unclassified devices remain, it repeatedly picks one arbitrarily, classifies it IN the MIS, and classifies adjacent devices OUT. Far more sophisticated centralized algorithms exist, but let's focus instead on a way for devices to classify themselves, based solely on local communication, without an all-knowing central solver.

\section{TALKDOWN}

The decentralized MIS algorithm, called TalkDown, operates asynchronously: Devices exchange messages, and each device eventually classifies itself. TalkDown leverages the fact that wireless devices have unique IDs, or names; that any two devices capable of communicating know each other's names; and that names can be compared [e.g., numerically or lexicographically]. Each device creates two unordered sets, $\mathrm{HI}$ and LO, containing adjacent device names that compare respectively greaterlless than its own name.

To compute an MIS, each device first enters a "ListenUp" phase in which it collects messages from peer devices in its HI set. If it receives "I'm OUT" messages from every member of its HI set, then a device declares itself IN, but if it hears "I'm IN" from any HI peer, it declares itself OUT. It then enters a "TalkDown" phase that notifies all devices in its LO set of its decision. Devices with empty HI sets start the ball rolling by immediately declaring themselves IN and telling their Lo peers, "I'm IN." 


\section{FIGURE 2: EXAMPLE TOPOLOGY}

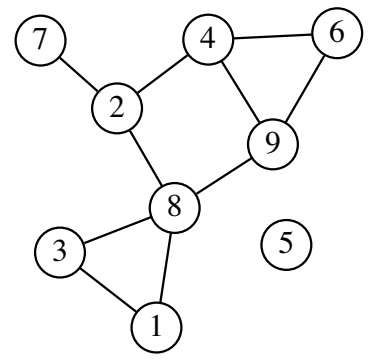

In the example topology of figure 2, devices 5, 7, and 9 have empty HI sets, so they skip the ListenUp phase, declare themselves IN the MIS, and announce their decisions to their Lo peers. This causes devices 2, 4, 6, and 8 to receive "I'm IN" messages during their ListenUp phases, so they declare themselves OUT. Device 3 receives an "I'm OUT" message from the sole device in its HI set, device 8, during its ListenUp phase, so it declares itself IN, enters its TalkDown phase, and sends "I'm IN" to device 1. Device 1 declares itself OUT. At this point all of the devices have classified themselves either IN $[3,5,7$, and 9) or OUT $(1,2,4$, 6, and 8].

Figure 3 shows the stages in which devices classify themselves and inform their peers. Solid circles and arrows indicate devices that end up IN the MIS and the "I'm IN" messages they send; dashed circles and arrows indicate OUT. Devices 5, 7, and 9 classify themselves 


\section{FIGURE 3: CLASSIFICATIONS AND MESSAGE PROPAGATION}
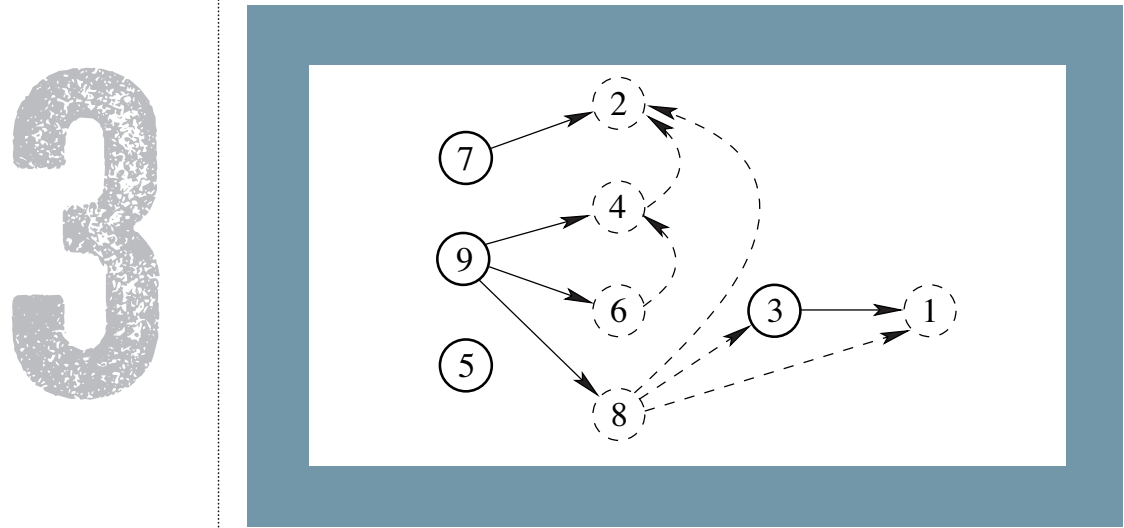

immediately. Devices 2, 4, 6, and 8 can classify themselves after receiving a single message. Device 3 must wait for 8 to classify itself, and 1 must wait for 3 .

Worst-case performance for the TalkDown algorithm, in terms of time to classify all devices, occurs when device names and network topology conspire malevolently. Consider the network (a)-(b)-(c)- - - (x)-(y)- (2). TalkDown messages propagate from (z) to a in this starcrossed topology, one after the other. Fortunately, as you would expect, the worst case is neither common enough nor bad enough to rule out TalkDown.

TalkDown has several nice properties.

\section{decentralization}

given a problem where the inputs are scattered solve it scatter-brained
It's simple and easy to implement, and it's frugal with communication: Exactly one bit of information travels between each pair of adjacent devices. TalkDown preserves privacy in the sense that no device learns anything it didn't already 
know beyond the final IN/OUT classification of adjacent devices-which it might learn or infer anyway if an MIS were computed by other means.

In some practical wireless networking applications, a new and different MIS must be computed from time to time. TalkDown can handle this requirement. Every device can replace its own name and the names of all adjacent devices with temporary pseudonyms used only for the purpose of computing a new MIS. For example, each pseudonym might be fashioned as a hash of the corresponding real name concatenated with a salt string. The times at which TalkDown must be run and the salts for making pseudonyms can follow a convention known to all devices, or in the scenario of figure 1, the base station can provide them.

\section{OTHER APPLICATIONS}

Decentralization isn't just for wireless networks, nor is TalkDown.

Turning from wireless networks to social networks, we find strong motivations for decentralized computing. Centralized solutions to social-networking problems raise anxiety over the concentration of power that inevitably accompanies concentration of knowledge. ${ }^{7}$ But social networking problems inherently require divulging all relevant information to a central solver-don't they?

Not always. Imagine a community that wants to throw a "mixer" party where strangers can meet: Anyone may attend so long as no two partygoers know one another. It's easy to see that a maximal independent set of the community's acquaintance network should attend. 
Individuals can invitelexclude themselves by executing TalkDown, obviating the need for everyone to disclose their acquaintances to a central solver. Luddites may execute TalkDown using pencil, paper, and postcards, though a simple software implementation such as the mixer.py script provided at the end of this column is more convenient. To hold a mixer party every Saturday with different invitees each time, replace all names with pseudonyms for the purpose of computing the MIS as described earlier. For example, concatenate each real name with the date of the party and compute a hash, as in

\$ echo 'John Smith 31 October 2020' | sha256sum 0c1fcd5c3a822559f32fbfc8951a5d712e9aa241a6b0279fa2feda9d6dd3e415

GOING FURTHER

Decentralized computing is a large topic, and this episode of Drill Bits has drilled a mere pilot hole, so to speak. Inquisitive readers can drill down further on their own, check out my example code, and try the suggested exercises.

Research literature has explored several decentralized computing models and paradigms. The quintessential abstract model is cellular automata (e.g., Conway's Game of Life, made famous by screen savers in the $1990 \mathrm{~s}^{13}$. The Game of Life is Turing-complete and therefore can in principle perform any computation-though seldom conveniently. "Market-oriented programming," a general paradigm intended for practical use, involves local computations that interact via auction-like mechanisms to solve optimization problems. ${ }^{12}$ Similar ideas later 
appeared with different motivations and emphases in the literature on "algorithmic mechanism design.",,11 Finally, some algorithms advertised as "distributed" are also decentralized or can be made so with modest effort; hunting for such algorithms can be fun.

\section{Bits}

Example code for this column is at https://queue.acm.orgl downloads/2020IDrill_Bits_02_sample_code.tar.gz. The tarball contains two MIS programs. The Python program mixer.py is decentralized, distributed, and parallel; the $C$ program mis.c is none of these. Shell scripts show how I run the two implementations on the example network of figure 2; one calls a separate script to check that the output is indeed an MIS.

Contrasting the two MIS programs highlights the tradeoffs between decentralized and centralized approaches. For example, the centralized solver can get away with storing edges in one direction only, sometimes cutting the memory footprint nearly in half. The decentralized implementation would be prettier were it not for fussy networking code and jumping through hoops to ensure that various write()s are atomic; see the comments in the shell script for details.

\section{Drills}

Ambitious readers, grab the sample code tarball and unpack it. Here are a few questions to ponder and exercises in ascending order of difficulty:

1. The mixer.py program uses lists for HI and LO. Would sets or dictionaries be better (e.g., more efficient) when 
checking for duplicates?

2. Would SOCK_RDM be better than the socket type that mixer.py currently uses?

3. Modify mixer.py to classify itself immediately if it receives an "I'm IN" message from a HI peer. Conduct tests on large topologies to quantify the reduction in time for all processes to classify themselves.

4. (Theory) Given a topology and one of its MISes, is it always possible to rename nodes so that the TalkDown algorithm computes the given MIS?

5. Modify mis.c to "stop when done"3 (i.e., terminate when the last classification happens]. Measure the performance improvement. How many unnecessary messages are sent by mixer.py?

6. Implement a fault-tolerant distributed TalkDown (hint: try building it atop Ken ${ }^{15}$.

7. Organize a mixer party using TalkDown. Which is harder, preparing the inputs or running the algorithm?

\section{Acknowledgments}

Timothy Chow and ACM FellowISIAM Fellow Robert Schreiber reviewed early drafts of this work, and Antonio Lain reviewed the example programs; all three provided valuable feedback.

\section{References}

1. Coatta, T.2008. From here to there, the SOA way. acmqueue 5(6); https:/lqueue.acm.org/detail. cfm?id=1388788.

2. Feigenbaum, J., Shenker, S. 2002. Distributed algorithmic mechanism design: recent results 
and future directions. In Proceedings of the Sixth International Workshop on Discrete Algorithms and Methods for Mobile Computing and Communications [September], 1-13; https:/ldoi.org/10.1145/570810.570812.

3. Kelly, T. 2020. Efficient graph search. acmqueue 18[4]; https://queue.acm.org/detail.cfm?id=3424304.

4. Killalea, T. 2016. The hidden dividends of microservices. acmqueue 14(3); https:/lqueue.acm.org/detail. cfm?id=2956643.

5. Lee, J. G., Cheung, G., Lee, S.-L., Sharma, P. 2013. Channel assignment for a wireless network. U.S. Patent \# 8,531,956 8,531,956 [September].

6. Natarajan, K. S. 1993. Robust scheduling mechanism for efficient bandwidth usage in multicell wireless local networks. U.S. Patent \# 5,210,753 (May).

7. Onion News Network. 2011. CIA's “Facebook” program dramatically cut agency's costs; https:/lwww.youtube. com/watch?v=ZJ380SHZvYU and https://www.theonion. com/cias-facebook-program-dramatically-cut-agencyscosts-1819594988.

8. Perlman, R. 1999. Interconnections: Bridges, Routers, Switches, and Internetworking Protocols, second edition. Addison-Wesley. Chapter 12.

9. Schreiber, R. S., Kelly, T. P. 2014. Determination of maximal independent sets of mobile devices. U.S. Patent \# 8,849,325 [September).

10. Tay, B. H., Ananda, A. L. 1990. A survey of remote procedure calls. ACM SIGOPS Operating Systems Review 24(3): 68-79;

https://doi.org/10.1145/382244.382832.

11. Varian, H. R. 2008. Designing the perfect auction. 
Communications of the ACM 51[8], 9-11; https:/ldoi. org/10.1145/1378704.1378708.

12. Wellman, M. P. 1996. Market-oriented programming: some early lessons. In Market-Based Control: A Paradigm for Distributed Resource Allocation. Chapter 4. World Scientific; http:I/strategicreasoning.org/wpcontent/uploads/2010/03/mbc95.pdf.

13. Wikipedia. Conway's Game of Life; https:Ilen.wikipedia. org/wiki/Conway's_Game_of_Life.

14. Wikipedia. Maximal Independent Set. https:/l en.wikipedia.org/wiki/Maximal_independent_set.

15. Yoo, S., Killian, C., Kelly, T., Cho, H. K., Plite, S. 2012. Composable reliability for asynchronous systems. In Proceedings of the Usenix Annual Technical Conference (June); https:I/www.usenix.org/system/files/conferencel atc12latc12-final206-7-20-12.pdf.

Terence Kelly (tpkelly@acm.org) is a Distinguished Member and a Lifetime Member of the ACM. He studied computer science at Princeton and the University of Michigan followed by 18 years as an industrial researcher (HP Labs) and software engineer. Scatterbrained computing is his favorite kind.

Copyright $\odot 2020$ held by ownerlauthor. Publication rights licensed to ACM. 